The Astrophysical Journal, 178:701-706, 1972 December 15

(C) 1972. The American Astronomical Society. All rights reserved. Printed in U.S.A.

\title{
SEARCH FOR CORONAL LINE EMISSION FROM THE CYGNUS LOOP
}

\author{
D. W. Kurtz ANd P. A. VANDEn Bout \\ University of Texas at Austin and McDonald Observatory \\ AND \\ J. R. P. ANGEL* \\ Columbia Astrophysics Laboratory, Columbia University \\ Received 1972 June 30
}

\section{ABSTRACT}

The flux from the edges of the Cygnus Loop in the coronal line [Fe XIV] $\lambda 5303$ is measured to be less than $5 \times 10^{-9}$ ergs $\mathrm{cm}^{-2}$ sterad $^{-1} \mathrm{~s}^{-1}(0.017 \mathrm{R})$ in a $3 \AA$ band centered on the line. This upper limit is an order of magnitude lower than the predicted total flux in the line and implies that there must be substantial broadening of the line profile by mass motion or that the temperature is somewhat higher than the present best estimate from X-ray data.

\section{INTRODUCTION}

The Cygnus Loop is a strong source of long-wavelength $(\lambda>10 \AA) \mathrm{X}$-rays identified by Grader, Hill, and Stoering (1970) and studied further by Gorenstein et al. (1971) and Bleeker et al. (1972). X-ray emission is extended over the region of about $3^{\circ}$ in diameter shown by the optical filaments, with an intensity distribution indicative of a shell structure. The most accurate spectral data by Bleeker et al. show a thermal X-ray spectrum, with a plasma temperature $T=2.7 \pm 0.4 \times 10^{6}{ }^{\circ} \mathrm{K}$. This is consistent with the data of Gorenstein et al. which were initially fitted with a simple exponential spectrum having $T=4 \times 10^{6}{ }^{\circ} \mathrm{K}$ and a single emission line of $\lambda=19 \AA$. Tucker (1971), analyzing the same data, estimates that the best fitting optically thin plasma might well be lower, $T \simeq 2 \times 10^{6}{ }^{\circ} \mathrm{K}$. Recently Stevens, Garmire, and Riegler (1972) have confirmed the thermal emission spectrum and find strong evidence of line emission. The identification and further study of X-ray emission lines, which could give valuable information about physical conditions in the plasma, are difficult because Bragg crystal and grating techniques cannot easily be applied to a diffuse source of radiation. However, the possibility arises that the same information can be derived from ground-based observations.

If the X-rays are indeed emitted by a hot plasma with a temperature of a few million degrees, then, as Shklovskii (1967) has argued, optical coronal-line emission should also be produced. The observed temperature is such that diffuse emission of [Fe XIV] $\lambda 5303$ can be expected from the same regions producing X-ray emission. If this emission were detectable against the background of starlight, then the high-temperature regions could be mapped, and physical parameters such as expansion velocity could be determined.

\section{ESTIMATED STRENGTH AND PROFILE OF CORONAL LINES}

The relative strength of thermal X-ray emission and coronal line emission from an optically thin plasma depend only on the temperature distribution and abundance of

* Alfred P. Sloan Research Fellow. 


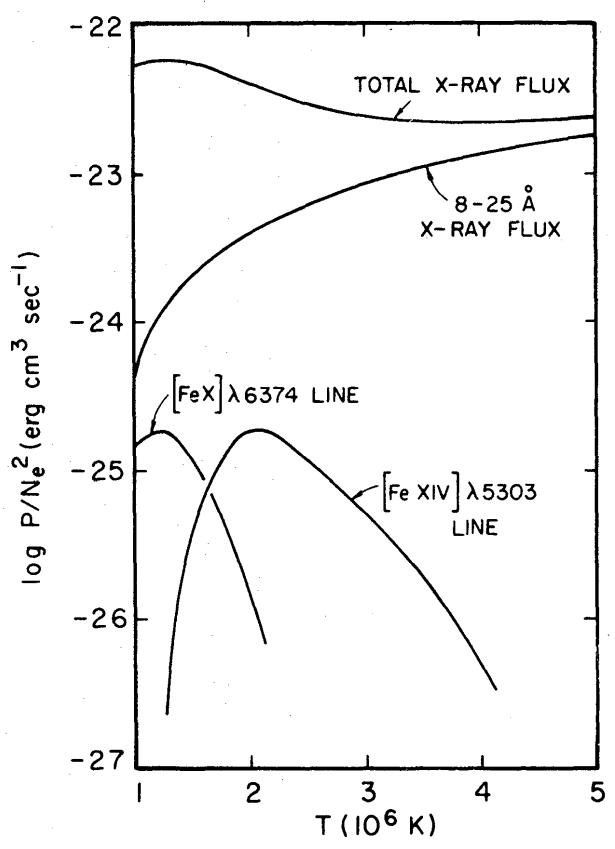

Fig. 1.-Power radiated in X-rays and coronal lines as a function of temperature

elements, and estimates of the total X-ray flux from thin plasmas have been made by several authors. It is convenient to use the recent results of Tucker and Koren (1971), shown in figure 1, which are for solar abundance. The power expected in the green ([Fe XIV] $\lambda 5303$ ) and red ([Fe X] 26374$)$ coronal lines is also shown in figure 1. Here we have adopted the emissivities calculated by Nussbaumer and Osterbrock (1970) and assumed that $\log \left(N_{\mathrm{Fe}} / N_{\mathrm{H}}\right)=7.5-12$, yielding the ratio $N_{\mathrm{Fe}} / N_{e}=2.6 \times 10^{-5}$. It will be seen that at the most favorable temperatures, $2.1 \times 10^{6}{ }^{\circ} \mathrm{K}$ for [Fe XIV] $\lambda 5303$ and $1.2 \times 10^{6}{ }^{\circ} \mathrm{K}$ for $[\mathrm{Fe} \mathrm{X}] \lambda 6374$, the power in coronal lines is only a few hundred times less than the total X-ray power, and so the two photon fluxes are comparable. The total X-ray flux obtained by both Gorenstein et al. (1971) and Bleeker et al. (1972) is $2 \times 10^{-8}$ ergs $\mathrm{cm}^{-2} \mathrm{~s}^{-1}$ and is insensitive to the choice of temperature. Adopting this value, we can with the aid of figure 1 calculate the coronal line flux for any assumed single temperature or distribution. If the plasma temperature is $2.7 \times$ $10^{6}{ }^{\circ} \mathrm{K}$, as indicated by the X-ray data, then the predicted total flux in $\lambda 5303$ is $6 \times$ $10^{-11}$ ergs cm ${ }^{-2} \mathrm{~s}^{-1}$. Spread uniformly over the whole nebula, which we take to be an annular region of inner radius $2^{\circ}$ and outer radius $3^{\circ}$, the corresponding surface brightness is $4.8 \times 10^{-8}$ ergs $\mathrm{cm}^{-2}$ sterad ${ }^{-1} \mathrm{~s}^{-1}$, that is, 0.16 Rayleighs.

The practical difficulty in detecting the optical coronal lines arises because of the relatively bright continuum background of starlight from the Galaxy and because the line profiles will be broadened by thermal and mass motions. The width from thermal Doppler broadening at $3 \times 10^{6}{ }^{\circ} \mathrm{K}$ is $1 \AA$ (FWHM) for $\lambda 5303$. Mass motions in the remnant are a more serious potential source of broadening. If the plasma is indeed heated by a shock of velocity $500 \mathrm{~km} \mathrm{~s}^{-1}$, as suggested by Tucker (1971), then maximum shifts of $\pm 10 \AA$ can be expected. Taking a simple model of an expanding spherical shell, the observer would see the outline of the shell as a ring of maximum brightness and small radial velocity. However, allowing for the finite thickness of the shell and deviations from a perfect sphere, it is apparent that broadening of $5 \AA$ (FWHM) or more could arise, even in the bright ring region. 


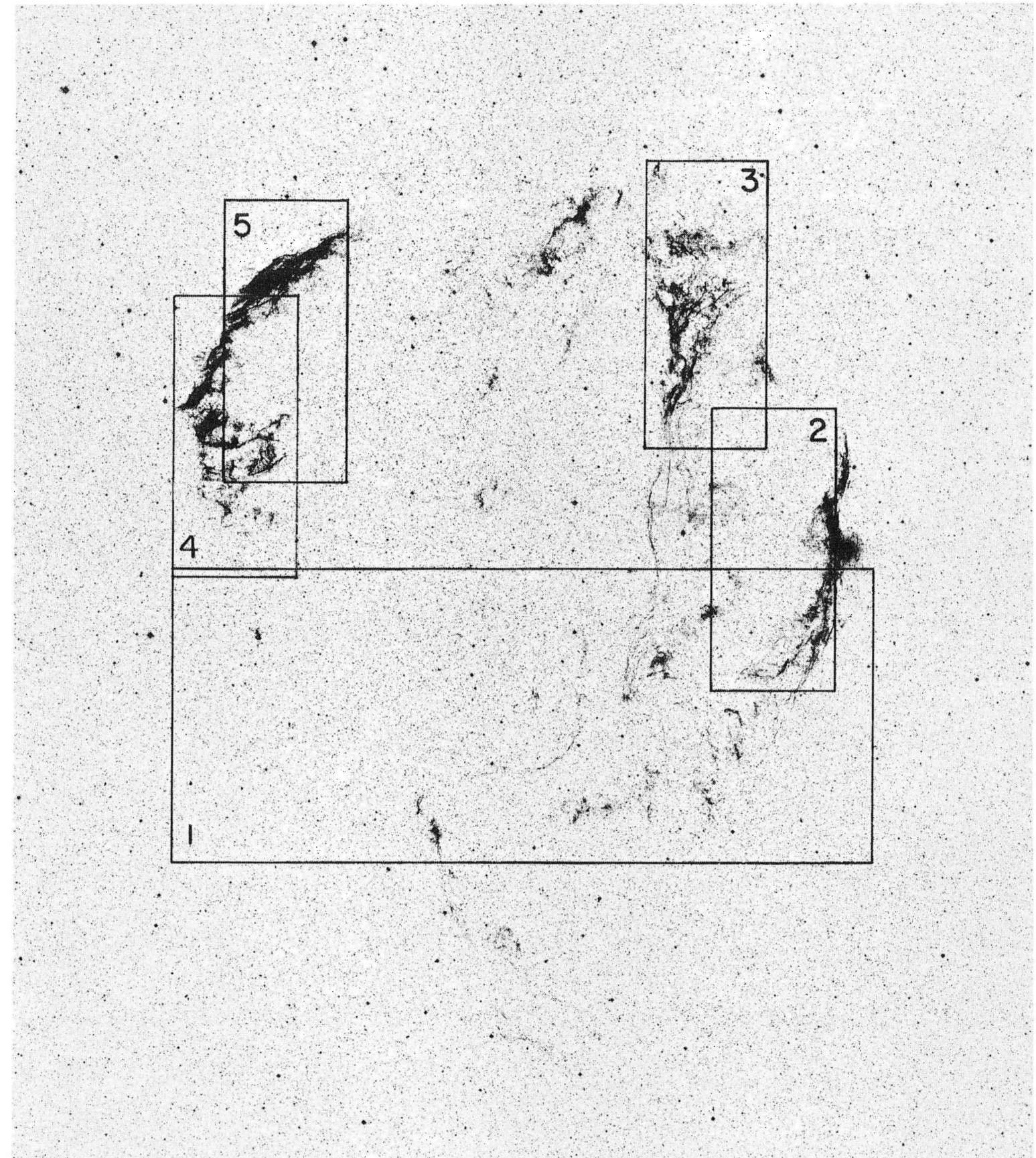

FIG. 2.-Areas and wavelengths observed in the Cygnus Loop; position 1, $\lambda 6374$; positions 2-5, $\lambda 5303$. 


\section{INSTRUMENT AND OBSERVATIONS}

An instrument was made with interference filters, which are especially useful when diffuse radiation is to be analyzed for one or two relatively broad spectral features. It consists of an objective lens of either 50 or $125 \mathrm{~cm}$ diameter with an aperture stop in the focal plane which limited the field of view to $90^{\prime} \times 195^{\prime}$ and $33^{\prime} \times 77^{\prime}$, respectively. The light is collimated by a second lens, passed through a $5.1-\mathrm{cm}$ diameter interference filter, and refocused by a third lens onto a photomultiplier tube cathode. The interference filter is enclosed in an isothermal housing of controlled, variable temperature, which allows the central bandpass wavelength to be tuned exactly to the coronal line wavelengths. The filter could be rocked by an electromagnet between the two positions, one normal to the optical axis and the other tilted such that the central bandpass wavelength was shifted $4 \AA$ to the blue. Narrow filters were obtained for both the green and red lines and were calibrated at the coudé scanner of the 107inch $(272-\mathrm{cm})$ telescope at McDonald Observatory, giving the temperature dependence of central wavelength and the bandpass. These were $3.2 \AA$ (FWHM) for the $\lambda 5303$ filter and $2.5 \AA$ (FWHM) for the $\lambda 6374$ filter. In operation the filter is maintained at the correct temperature for maximum transmission of the coronal lines in the normal position and switched periodically $(1 \mathrm{~Hz})$ to the tilted position. The phototube pulses are gated synchronously into two scalers.

Observations were made with the $5303 \AA$ filter of the four smaller areas in the Cygnus Loop shown in figure 2 . For each area an integration was made to determine the counting rate for the two filter positions, and this was compared with a similar integration on a region with similar density of background stars a few degrees out of the remnant. Any difference in the two spectra would then show as different ratios in the two scalers. Frequent observations of the nearby bright star $\alpha$ Cyg were used to convert the counting rates to absolute fluxes incident at the Earth. Table 1 lists for each of these regions in the Loop the excess flux in a band centered on $5302.9 \AA$ and also for the large region (1) in a band centered at $6374.5 \AA$. The excess is expressed as a fraction of the mean flux in the bands due to sky background, and as an absolute flux in ergs $\mathrm{cm}^{-2}$ sterad $^{-1} \mathrm{~s}^{-1}$. The quoted errors are the standard deviation calculated from counting statistics alone. It will be seen that in no position is there any significant increase in the background continuum at the coronal-line wavelength. In the $3 \AA$ band at $5303 \AA$ where most of the data are taken, the fractional increment in the background continuum is no more than a percent or so, at least relative to the flux at $5299 \AA$.

From the tabulated values of absolute flux we deduce that for the best observed positions, 2 and 3, an upper limit $(3 \sigma)$ of the excess in the $\lambda 5303$ band is $5 \times 10^{-9}$ ergs $\mathrm{cm}^{-2}$ sterad $^{-1} \mathrm{~s}^{-1}$. This upper limit is an order of magnitude less than the best estimate above of $4.8 \times 10^{-8} \mathrm{ergs} \mathrm{cm}^{-2}$ sterad $^{-1} \mathrm{~s}^{-1}$ for the line flux. The discrepancy can be explained if either there is rather more Doppler broadening than expected, if the temperature is higher than assumed, or if the iron abundance is low. For linewidths

TABLE 1

Excess Flux in Regions in the Cygnus Loop

\begin{tabular}{|c|c|c|c|}
\hline Position & $\lambda(\AA)$ & $\begin{array}{l}\text { Relative Fractional } \\
\text { Excess Flux } \\
\text { (percent) }\end{array}$ & 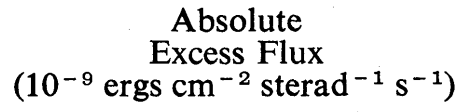 \\
\hline $\begin{array}{l}1 \ldots \ldots \ldots \ldots \\
2 \ldots \ldots \ldots \ldots \ldots \\
3 \ldots \ldots \ldots \ldots \\
4 \ldots \ldots \ldots \ldots \ldots \\
5 \ldots \ldots \ldots \ldots \ldots\end{array}$ & $\begin{array}{l}6374 \\
5303 \\
5303 \\
5303 \\
5303\end{array}$ & $\begin{array}{l}-1.8 \pm 1.3 \\
-0.34 \pm 0.50 \\
+0.31 \pm 0.60 \\
+1.6 \pm 1.1 \\
-2.2 \pm 1.6\end{array}$ & $\begin{array}{l}-8.4 \pm 6.1 \\
-1.1 \pm 1.6 \\
+1.1 \pm 2.2 \\
+5.2 \pm 3.7 \\
-6.9 \pm 5.2\end{array}$ \\
\hline
\end{tabular}


greater than the filter width of $3 \AA$, the sensitivity is reduced because not all the energy falls in the bandwidth. A further reduction occurs if the line is so wide that a significant amount of energy falls in the comparison channel $4 \AA$ away. The shock-wave model, summarized in a recent review by Woltjer (1972), yields a shock velocity $V=0.263$ $T^{1 / 2}=430 \mathrm{~km} \mathrm{~s}^{-1}$ when $T=2.7 \times 10^{6}{ }^{\circ} \mathrm{K}$. If the emission is from a spherical shell expanding uniformly with this velocity, then, in regions 2 and 3 at the edge of the shell, lines of width $\sim 5 \AA$ are predicted, and about half the line flux would appear in the $3 \AA$ band. More severe broadening or shifting of the line center is required to explain the low limit to the observed flux, but this could easily arise if the expansion is not very uniform. Alternatively, we note from figure 1 that the $\lambda 5303$ emission drops off quite steeply with increasing temperature. If the plasma temperature is really $3.5 \times$ $10^{6}{ }^{\circ} \mathrm{K}$, which is the upper limit obtained by Bleeker et al., then a surface brightness of $6 \times 10^{-9} \mathrm{ergs} \mathrm{cm}^{-1}$ sterad ${ }^{-1} \mathrm{~s}^{-1}$ is predicted, consistent with our data if there is only little Doppler broadening.

This work was supported by the National Aeronautics and Space Administration under grants NGR 33-008-102 and NGR 44-012-209 and under a special grant; by the Research Corporation; and by the National Science Foundation under grant GP31356 X. We are grateful to Drs. G. Garmire and J. Bleeker for providing data prior to publication. This is Columbia Astrophysics Laboratory Contribution No. 66.

\section{REFERENCES}

Bleeker, J. A. M., Deerenberg, A. J. M., Yamashita, K., Hayakawa, S., and Tanaka, Y. 1972, preprint.

Gorenstein, P., Harris, B., Gursky, H., Giacconi, R., Novick, R., and Vanden Bout, P. 1971, Science, 172, 369.

Grader, R. J., Hill, R. W., and Stoering, J. P. 1970, Ap. J. (Letters), 161, L45.

Nussbaumer, H., and Osterbrock, D. E. 1970, Ap. J., 161, 811.

Shklovskii, I. S. 1967, Soviet Astr.-AJ, 10, 594.

Stevens, J., Garmire, G., and Riegler, G. 1972, private communication.

Tucker, W. 1971, Science, 172, 372.

Tucker, W., and Koren, M. 1971, Ap. J., 168, 283.

Woltjer, L. 1972, Ann. Rev. Astr. and Ap., to be published, August, 1972. 